\title{
Persönliche und methodische \\ Wechselwirkungen
}

Haltungen, Erfahrungen und persönliche Herangehensweisen auf Arzt- und Patientenseite spielen genauso eine Rolle wie die jeweiligen Behandlungsmethoden, die mit dem Ziel der Heilung eingesetzt werden $=$ Ein persönlicher Kommentar

Helmut Milz

\section{Vorbemerkung}

Im folgenden Beitrag möchte ich, auf Basis meiner vier Jahrzehnte an klinischer Praxis und verschiedenen Lehrtätigkeiten, einige Gedanken zur Diskussion stellen. Ich erhebe dabei keinen durchgängig wissenschaftlichen Anspruch. Es geht mir darum anzuerkennen, dass Ärzte und Ärztinnen die gleiche Problemlage eines erkrankten Menschen aus ganz unterschiedlichen, manchmal auch aus nicht umstandslos kompatiblen Blickwinkeln betrachten und behandeln. Dieses Vorgehen erfordert eine sensible und erfahrene Verbindung von Heilkunde und Heilkunst, damit wir zusammen mit unseren Patientinnen und Patienten das für sie Wünschenswerte finden und sie nicht mit dem maximal Machbaren unnötig überfordern. Dazu kann auch eine Reflexion darüber hilfreich sein, wie die individuellen Motive von ÄrztInnen deren jeweilige Präferenz für Methoden beeinflussen können.

\section{Leibgedächtnis, Erinnerungen und Vorstellungen}

Zur Frage, was der Körper, was das Lebendige wirklich sei, schreibt der Medizinhistoriker Philipp Sarasin [12]: Es „scheint keine lösbare Frage zu sein; entscheidend ist daher, welches Bild - in einem Text, als visuelle Abbildung oder als Inszenierung - wir uns von unserem Körper machen, von dem wir sagen, es sei unserer“.

Unsere Erinnerungen, Vorstellungen und Gefühle wirken sich bekanntermaßen auf unser leibliches Befinden und die Physiolo-

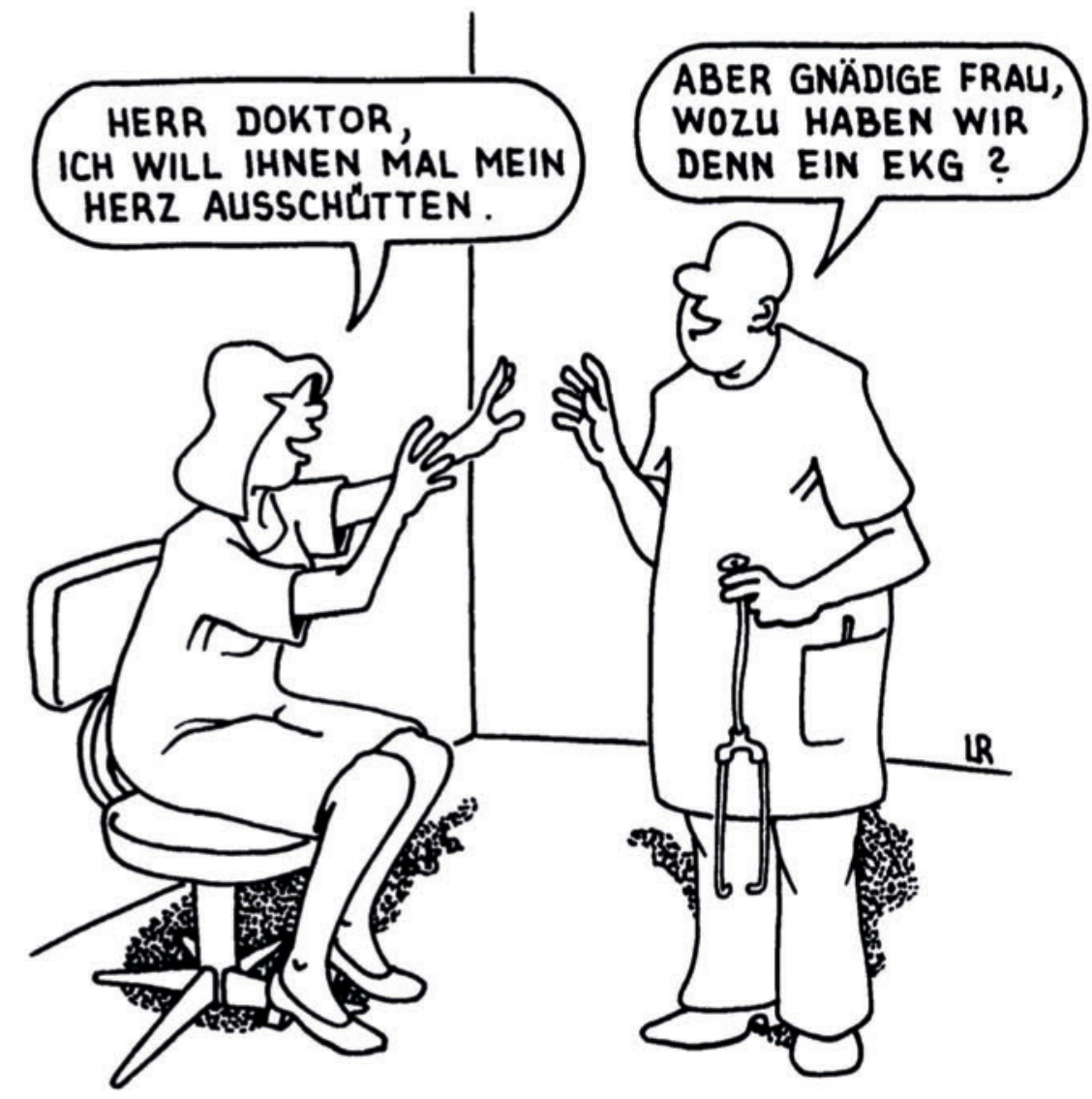

Abb. 1 Quelle: Reinhold Löffler/Cartoon-Archiv

gie unseres Körpers aus. Dies macht sich u.a. in veränderten Muskelanspannungen, Herzaktionen, Atemmustern, Verdauungsaktivitäten, Blasenaktionen, Blutdruckverände- rungen, hormonellen oder immunologischen Reaktionen bemerkbar - sofern wir diese beachten. Die Stressforschung hat dazu seit Jahrzehnten vielfältige Labordaten geliefert. 


\section{Zusammenfassung}

Die Methoden und Herangehensweisen an eine Heilung sind individuell und nicht immer linear. Persönliche Haltungen und Erfahrungen sowie zahlreiche kontextuelle Faktoren beeinflussen den Weg der Heilung genauso wie die jeweils eingesetzten

Therapiemethoden. Dabei geht es um eine respektvoll angemessene Auswahl von Wünschenswertem und Machbarem. Das Erkennen der Problemlage des erkrankten Menschen erfordert ein sensibles Vorgehen, gerade bei den immer häufiger auftretenden sog. funktionellen bzw. medizinisch nicht eindeutigen Erkrankungen.

Eine Reflexion über die gewählte Sprache, Zuhören, die eigenen Motive für eine Methodenpräferenz können hilfreich sein, um gemeinsam mit den Patienten das für sie Wünschenswerte zu finden und sie nicht mit dem maximal Machbaren zu überfordern

Der Autor stellt ausgewählte Aspekte aus seiner jahrzehntelangen klinischen Praxis und Lehrtätigkeit zur Diskussion.

Bei Menschen, die unter Angststörungen leiden, können schon Gedanken an einen drohenden Kontrollverlust, in ihrem „Leibgedächtnis“ ähnliche Empfindungen von Übelkeit, Herzstolpern, Schwindelgefühl, Atemnot etc. auslösen, wie sie in früheren Krisen erlebt wurden. Im Rahmen einer Posttraumatischen Belastungsstörung können spontane, sog. „Flashbacks“ (plötzlich einschießende Erinnerungen an frühere Szenen) unerwartete panikartige Zustände auslösen. Die anfangs noch hilflosen Versu- che dieser Menschen, ihre Erinnerungen und Empfindungen zu unterdrücken, können möglicherweise die Symptome noch verstärken. Insgesamt kann dies zu dem führen, was man in der Psychotherapie die „Angst vor der Angst“ nennt.

In der anamnestischen Aufarbeitung von Ängsten glauben viele Experten ein eindeutiges „Ereignis oder Datum“ als „Beginn oder Auslöser“ identifizieren zu können - etwa ein Unfall, ein plötzlicher Verlust von nahestehenden Menschen, ein traumatisches Geburtserlebnis, ein gewaltsamer oder sexueller Übergriff. Solche markanten Ereignisse können auch singulär verheerende, langfristige Konsequenzen haben. Es ist aber ebenfalls möglich, dass die Heftigkeit ihrer gezeigten Reaktionen die Konsequenz einer länger zurückreichenden psychologischen Disposition ist. In diesen Fällen kann es sein, dass jemand in seiner frühen kindlichen Entwicklung z.B. kein sicheres Bindungsverhalten erleben konnte, dass frühe Trennungsängste oder schwere Verluste ihn/sie sprachlos zurückgelassen haben oder dass er/sie durch die starken Ängste einer überfürsorglichen Mutter geprägt wurde. Danach kann ein neues stark gefühlsbeladenes Ereignis zu einem massiven Ausbruch der lange latent vorhandenen Ängste führen. Frühere Erlebnisse eines Menschen können seine zukünftige Erwartungshaltung wesentlich prägen.

\section{Ein Beispiel aus der Praxis}

Der Vater eines Patienten verstarb plötzlich im Alter von 40 Jahren an einem Herzinfarkt. Als sich der Patient selbst dem gleichen Alter seines verstorbenen Vaters nähert, erlebt er eine erhebliche existenzielle Verunsicherung. Er hat zudem erfahren, dass er möglicherweise „erblich vom Vater belastet sei“. Solche „fixierten Erwartungen“ können bewirken, dass ein Patient „plötzlich“ ähnliche Symptome eines „Herzinfarkts“, wie Engegefühle in der Brust, stechende Schmerzen oder Atemnot verspürt. Er ruft in seiner Angst einen Notarzt zu Hilfe.

Auch wenn ihm anschließend Ärzte in der Notaufnahme nach erfolgten Untersuchungen durch Labor und EKG die objektive „Entwarnung“ mitteilen („Es ist alles bestens bei Ihnen, Ihnen fehlt zum Glück nichts“), hört dieser die Botschaft trotzdem „eigen-sinnig“ und mit „seinen Ohren“. Er mag kurzfristig erleichtert sein, aber seine Zweifel bleiben. Den Ärzten fehlen aufgrund der knapp bemessenen Zeit in der Ambulanz die notwendigen Kenntnisse der Hintergründe des Patienten.

Möglicherweise quälen den Patienten weiterhin ängstlich-verzweifelte Fragen, wie: „Was wäre, wenn meine Frau plötzlich ohne mich und allein mit den Kindern dastehen würde - so wie es damals meiner Mutter mit uns Kindern ergangen ist?" Infolge des frühzeitigen Verlusts seines Vaters hat er früh ein hohes Pflichtgefühl gegenüber seiner Mutter und den Geschwistern entwickelt. Er nahm sich schon immer vieles „zu Herzen“, öfter wurde es ihm „schwer ums Herz“. Zugleich hatte er immer „ein großes Herz" für die Sorgen von anderen. Heute geht die moderne Linguistik davon aus, dass die menschliche Sprachentwicklung wesentlich ihren Ursprung in körperlichen Erfahrungen hat und sich diese in der „Metaphorik der Sprache“ niederschlagen [8].

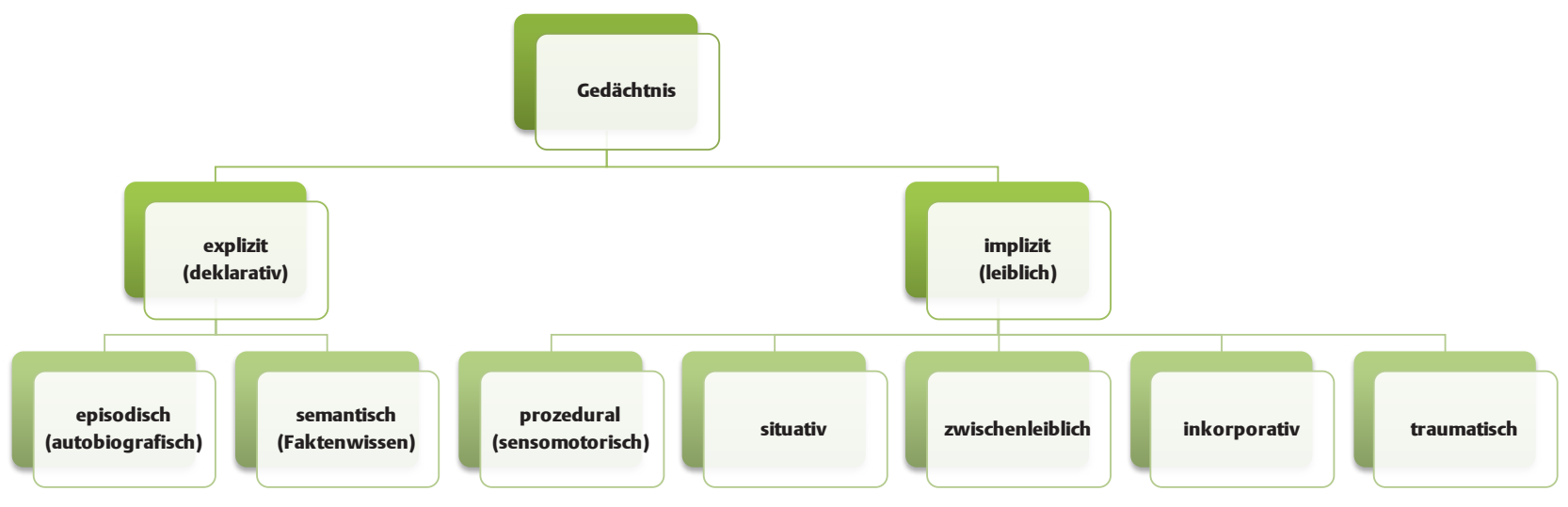

Abb. 2 Gedächtnissysteme im Überblick [5]. 


\section{Die moderne Linguistik geht davon aus, dass die Sprachentwicklung ihren Ursprung wesentlich in körperlichen Erfahrungen hat.}

Der Patient übertrug unbewusst seine aufopfernde Haltung auch in sein späteres berufliches Leben. Er arbeitete als Qualitätskontrolleur einer größeren Firma. Dort versuchte er, „alles zu erledigen, was man mir aufgetragen hat", und trank regelmäßig große Mengen Kaffee, „um leistungsfähig zu bleiben“. Seine häufigen Magenschmerzen, die bei großen Belastungen und Selbstzweifeln häufiger auftraten, versuchte er lange Zeit zu ignorieren. Über die Jahre wurde er angespannter und nervöser, hatte Konzentrationsmängel, wurde lärmempfindlich, bekam Tinnitussymptome und Schlafstörungen. Schließlich machte er häufiger Fehler, die er mühsam zu verstecken versuchte. Dann traten „plötzlich, wie aus heiterem Himmel“ Herzbeschwerden und panische Ängste auf. „Jetzt geht es mir genau wie damals meinem Vater“, schoss es ihm durch den Kopf. Er hatte seinen Vater immer vermisst und ihm aber gleichzeitig nie verzeihen können, dass er ihn und die Familie so früh „im Stich gelassen“ habe.

Trotz eines weitgehend unauffälligen EKG-Befunds bleiben beim Patienten bohrende Fragen ob seiner weiteren Behandlung und möglichen „Heilung“ offen. Wie kann er lernen, die „Zeichen und Botschaften“ (Symptome) seines Körpers anders und konstruktiver zu deuten? Kann es ihm gelingen, den bisher kaum betrauerten Verlust seines Vaters besser anzunehmen? Wie kann er sich in Zukunft weniger "perfekt“ in Arbeit und Familie aufopfern und sich dabei trotzdem selbst anerkennen als jemanden, der wertvoll ist und „sein Bestes versucht“?

Unterschiedliche Deutungsversuche und Hilfsangebote - welche Dosis kann helfen?

Dieser leicht modifizierte Fall aus meiner Praxis ist nur ein Beispiel für die sogenannten „funktionellen“ oder "medizinisch nicht eindeutigen" Erkrankungen: Sie machen epidemiologisch heute die Mehrzahl aller Arztbesuche in ambulanten Praxen aus. Bei diesen Leiden ergeben die Laboruntersu- chungen oder technisch-bildgebenden Verfahren keinen symptomadäquaten medizinischen Befund. Die ärztliche Feststellung: „Ihnen fehlt nichts“, ist aber nur scheinbar und kurzfristig beruhigend. Die weiterführende Frage: „Was ist Ihnen möglicherweise zu viel?“, wird aus Zeitnot in der Regel eher vermieden.

Je nach dem fachlichen Blickwinkel des Arztes bzw. der Ärztin, den oder die ein solcher Patient aufsucht, können seine Beschwerden und sein Leiden unterschiedlich verstanden und bewertet werden. Weiter oben habe ich, notwendigerweise verkürzt, eine psychosomatische Sichtweise des Geschehens beschrieben. Hinzu kommen pathophysiologische, genetisch disponierte oder vergleichende, klinische Aspekte. Aus einem gänzlich anderen Körperverständnis heraus, wie etwa der „energetischen Sicht“ der Traditionellen Chinesischen Medizin (TCM), könnte ein Behandler z. B. Dysbalancen zwischen Feuer- und Wasserelementen annehmen und dementsprechende Akupunkturinterventionen zur Regulierung des "Qi-Flusses“ einsetzen. Aus ernährungsmedizinischer Perspektive könnte dem Patienten vorgeschlagen werden, seine hohen Koffeinmengen zu reduzieren oder herzkräftigende Heilkräuterextrakte zu sich zu nehmen. Körpertherapeutisch könnte man Manipulationen seiner ängstlich bedingten Muskelverspannungen anraten. Atemtherapeutisch wäre das Erlernen einer bewussten Bauchatmung sinnvoll, um seine stressbedingt fixierte Thoraxatmung zu erweitern. Eine Verbesserung seiner Körperwahrnehmung und mehr Gelassenheit sowie die Einübung von Ruhepausen und Entspannungsverfahren erscheinen angeraten. Therapeutische Gespräche mit seiner Ehefrau könnten dabei helfen, bisher nicht ausgesprochene Ängste besser zu verstehen. Nicht zuletzt würden sozialmedizinische Beratungen über andere Möglichkeiten der Arbeitsgestaltung angebracht sein usw.

All diese Aspekte, welche sich jeder für sich auf „sinnvolle“ Hilfsangebote und Rat- schläge beziehen, könnten aber in ihrer Summe und ohne eine geschickte und geduldige Vermittlung bei der vorliegenden perfektionistischen Grundeinstellung des Patienten möglicherweise auch zusätzliche Belastungen für ihn mit sich bringen. Viele gut gemeinte, aber schlecht dosierte Ratschläge von „ganzheitlichen“ Ärzten und Therapeuten könnten ihn unbeabsichtigt dazu drängen, ein „ganzheitlicher Gesundheitsathlet“ zu werden. Möglicherweise würde ihn dann zusätzlich noch ein schlechtes Gewissen belasten, wenn er nicht regelmäßig alle Ratschläge befolgen würde. Die meisten Patienten „vergessen“ aber gute Ratschläge ihrer Ärzte so lange, bis wieder ein neues Problem auftaucht.

\section{Komplementär bedeutet für mich} mehr als die Addition von Möglichkeiten

Eine ausgewogene Integration von medizinischen und therapeutischen Optionen erfordert die Verbindung von Heilkunde(n) und Heilkunst. Die meisten Kolleginnen und Kollegen akzeptieren eine wichtige Grundfrage: „Warum hat dieser Mensch dieses Leiden zu diesem Zeitpunkt?“ Die Beantwortung dieser Frage erfordert jedoch Zeit. Man kann ihr auf der Basis verschiedener Konzepte unterschiedlich nachgehen. Alle Antworten bleiben jedoch nur annähernd und unvollständig. In den möglichen Antworten vermischen sich Kausalitäten und Plausibilitäten, trotz aller Bemühungen um Evidenz. Ständig gesellen sich neue Fragmente von Wissen hinzu, und neue Forschungsergebnisse hinterfragen, ergänzen oder verändern diese Antworten. Der englische Psychiater R. Hinshelwood schrieb einmal treffend: „Es ist das glorreiche Privileg des Akademikers zu wissen, daß sie auf dem Weg sind, alles zu wissen. Es ist das demütige Schicksal des Praktikers, zu wissen, daß fast alles unsicher und paradox bleibt“ [6]. Mit diesen alltäglichen Unsicherheiten seinen Patienten ehrlich und nicht beliebig zu begegnen ist eine schwierige, aber unvermeidliche Herausforderung.

Was Praktikerinnen und Praktiker manchmal „über-hören“ ist eine weitere unausgesprochene Frage, welche sich die hilfesuchenden Menschen auf der anderen Seite der Begegnung implizit stellen: „(Wie) kann ich diesem Doktor vertrauen? Schaut er nur auf meine Symptome oder nimmt auch er mich, als besonderen Menschen, wahr?“ 
Welche Antworten sich auf diese „Vertrauensfrage" finden lassen, darüber entscheidet das Verhalten des Arztes.

Schätzt ein Arzt den Blick auf die Daten und Befunde des Monitors auf seinem Schreibtisch höher ein als das ,augenscheinliche“ Befinden des Menschen, der ihm gegenübersitzt? Verlegt er sich ausschließlich auf das „Ab- und Aushorchen“ eines Körpers oder hat er auch offene Ohren für das, was dieser sagt? Kann er die Zwischentöne hören, welche dieser äußert oder auch das, was ihm der Patient möglicherweise verschweigt? Hören ist ein physiologisches Geschehen, Zuhören erfordert aufmerksame Zuwendung.
Meist wird heute von unterschiedlichen Heilmethoden oder Heiltechniken gesprochen. Mir erscheint es ebenfalls wichtig, auch über die möglichen Motive nachzudenken, welche Ärzte und Therapeuten bewusst oder unbewusst zu ihrer jeweiligen Methodenwahl veranlassen. Diese Frage beschäftigt mich auch deshalb, weil ich in den vergangenen Jahren häufig mit Kolleginnen und Kollegen, die sich in Krisen befunden haben, psychotherapeutisch gearbeitet habe.

Bereits im 16. Jahrhundert hat Paracelsus, der einerseits als Doyen der Naturheilkunde gesehen wird und in dessen Namen zugleich verdiente Schulmediziner alljährlich mit Medaillen versehen werden, eine

\section{Hören ist ein physiologisches Geschehen, Zuhören} erfordert aufmerksame Zuwendung.

Berührt ein Arzt ausschließlich und gezielt einzelne Körperregionen oder nimmt er dabei gleichzeitig respektvollen Kontakt mit dem Menschen auf? Hat er ein Gespür dafür, wie der andere Mensch auf seine Berührungen reagiert? Wertschätzt er seine eigenen Erfahrungen und seinen „Riecher“ für das jeweils Besondere der Situation oder des Menschen? Welche Rollen können und dürfen seine Ahnungen oder Intuitionen spielen? Kann er diese selbstkritisch in seine Diagnosefindung und Therapieplanung einbeziehen? Will er alles für die anderen lösen oder ist er an einer kooperativen Praxis interessiert? Hat er noch ein Verständnis von Selbstheilungsprozessen und den möglichen Ressourcen eines Menschen sowie davon, wie sich diese im Heilungsprozess angemessen nutzen und fördern lassen? Solchen und ähnlichen Fragen bin ich in meinem letzten Buch „Der eigen-sinnige Mensch“ [11] differenziert nachgegangen.

\section{Unterschiedliche Motive und Zugänge}

Der Medizinhistoriker Heinrich Schipperges konstatiert zur „Naturheilkunde“ als eine der vielfältigen Richtungen der Ganzheitsmedizin, dass dieser alte, ,modisch verwaschene Begriff“ einer möglichst differenzierten Klärung bedürfe, „als darin bereits die beiden fundamentalen Leitbilder jeder Heilkunst enthalten sind: die ,Natur' und das ,Heilen' [13]“.
Typologie der „Heiler“ aufgestellt, welche bis heute kaum an Aktualität verloren hat. Edward Whitmont hat diese kursorisch zusammengefasst [15]:

- Die „naturales“ versuchen sich durch Anwendung von unterstützenden, ausgleichenden oder kompensierenden Modalitäten den Krankheitszuständen von außen entgegenzustellen (dies betrifft sowohl naturwissenschaftlichallopathische Medizin als auch die meisten Methoden der Naturheilkunde). Es geht darum, symptomatische Behandlungen zu finden, z.B. um Krämpfe zu lösen, Fülle zu entleeren, Mangelerscheinungen durch den Ersatz von fehlenden Stoffen zu kompensieren, etwas durch das Gegenteil auszugleichen (dies jedoch gilt nicht für präventive, ernährungsbezogene oder hygienische Maßnahmen). Die „naturales“ widmen sich auch theoretisch der Genese der Störungen, wenden sich aber vorwiegend praktisch deren sekundären, organisch bereits manifesten Ergebnissen zu. Dies erscheint vor allem dann notwendig, wenn „keine Zeit“ da zu sein scheint, um auf die Wirkung von möglichen Selbstheilungskräften zu warten, sondern eine rasche Linderung der Beschwerden notwendig geworden ist oder von ihnen erwartet wird.

- Die „specifici“ widmen sich eher den oft unsichtbaren Dynamiken, welche den „in Krankheiten fixierten Störungen“ zugrunde liegen könnten. Sie wollen durch ihre Interventionen „Blockaden beseitigen“ und wieder „einen gesunden Lebensfluss bewirken“ (z.B. Traditionelle Chinesische Medizin, Akupunktur, Homöopathie, Ayurveda oder verschiedene leibtherapeutische Ansätze).

- Die „spirituales“ stehen in gewisser Tradition zu den „Schamanen“ und wollen den „Wurzel- und Kräutergeistern“ (Paracelsus) Einhalt gebieten, um dadurch den Druck von deren „angreifenden Feldern“ zu beseitigen und den Kranken neuen „Raum zum Atmen“ schaffen. Sie übernehmen oft stellvertretende Rollen für die Erkrankten.

- Die „characterales“ wirken durch „die Macht des Wortes“ und die „Kraft ihrer Heilerpersönlichkeit“ (dies entspricht den Methoden der Psychotherapie).

- Die „fideles“ heilen durch die „Wirkung des Glaubens" und die Hingabe an von ihnen verehrte „transpersonale Kräfte“. Es bestehen viele Übergänge und Kombinationsmöglichkeiten zwischen diesen idealtypischen Beschreibungen von Paracelsus. Sie umfassen das heute vorhandene Spektrum von „ganzheitsmedizinischen Idealen“ an „Körper-Seele-Geist“-Interaktionen. Es gilt aber für jeden „Heilertypus“ seine/ihre notwendige Bescheidenheit zu praktizieren, da kaum jemand alle genannten Facetten gleichzeitig beherrschen kann. Dementsprechend müssen alle ihre eigenen Stärken und Schwächen (an)erkennen und respektieren, wenn sie hilfreiche Kooperationen mit ihren Patienten und Patientinnen fördern wollen.

Neuere psychologische und soziologische Studien zur Motivation und Berufswahl von Therapeuten erweitern unser Verständnis für die persönlichen Charakteristika von Helferinnen und Helfern. Unterschiedliche Talente, wissenschaftliche Neugier, empathische Fähigkeiten, Altruismus, schwierige Familiensituationen, Vorbilder, bis hin zur Selbstverwirklichung oder Selbsthilfe können dabei wichtige Motive sein [3, 7]. Dabei lassen sich meist nur wenige lineare Erklärungen für die individuelle Methodenwahl finden.

Wenn ich meinen vielgestaltigen beruflichen Weg reflektiere, dann spielten dabei persönliche Erfahrungen, bewusste und unbewusste Motive, durchaus auch manche „Zufällige“ Begegnungen und ergriffene Möglichkeiten sowie eine anhaltende Lust zu lernen und kritisch zu hinterfragen un- 
terschiedliche Rollen. Neben meinem Respekt für die Leistungen der naturwissenschaftlich basierten Medizin interessieren mich auch deren Grenzen und Schwachstellen, vor allem, was ihre Möglichkeiten zur Gesundheitsförderung oder ihren Umgang mit chronischen Leiden angeht. Dies hat mich neugierig gemacht, auch von anderen Gebieten zu lernen: komplementäre Heilverfahren, TCM [9], Public Health und Gesundheitsförderung, Psychosomatik, Psychotherapiemethoden, Leibtherapien [10], philosophische Sinnfragen, die Geschichte der Heilkunde [11], transdisziplinäre Möglichkeiten des Austauschs, nicht zuletzt die Poesie der Sprache.

In meiner praktischen Arbeit ist es für mich wichtig, den jeweils passenden Behandlungs- und Handlungsweg für die unterschiedlichen Patientinnen und Patienten zusammen mit diesen zu finden, statt ihnen meine methodischen Präferenzen aufzudrängen. Dies bedeutet auch, dass ich sie manchmal an andere Kolleginnen und Kollegen weiterempfehle, die ihren individuellen Heilungswegen näherstehen.

\section{Was wirkt in Arzt-Patienten-Bezie- hungen?}

Wenn man sich die Wirkfaktoren von therapeutischen Beziehungen anschaut, dann lassen sich folgende Elemente finden. Am Anfang steht die Entwicklung einer stärker gefühlsbesetzten, vertrauensvollen Beziehung. Beide teilen ein gemeinsames „Glaubenssystem“ oder einen „Mythos“, der als Erklärung für die Ursachen von Krankheiten und deren mögliche Überwindung anerkannt wird. Es entsteht eine Atmosphäre, die es den Patienten ermöglicht, ihre Probleme so zu beschreiben, dass sie prinzipiell an deren Bewältigung und Lösung mitarbeiten können. Dadurch kann es gelingen, Perspektiven und Hoffnungen aufzuzeigen, welche helfen, die anfängliche Hilflosigkeit besser zu überwinden. Es werden Erfolgserlebnisse vermittelt, welche die Zuversicht und Kompetenzen der Patienten verbessern. Letztlich ermöglicht ihnen dies, quasi „am eigenen Leibe“ spür- und fühlbare Erfahrungen von Veränderung in ihren Haltungen, Einstellungen und Verhaltensweisen zu erleben [4].

\section{Kooperative Beziehungsgestal- tung und Placeboeffekte}

Wie es Ärzten gelingt, aus zufälligen Begegnungen mit fremden Menschen eine möglichst vertrauenswürdige Beziehung zu entwickeln, davon hängt, methodenunabhängig, die Wirkung ihrer Arbeit ab. Über lange Zeit wurden Placeboeffekte oder „Placeboantworten“ auch von Ärzten eher als Störfaktoren belächelt. Klinische und pharmakologische Forschungen haben in den letzten Jahren deutlicher gemacht, dass dieses psycho-sozio-biologische Phänomen auf vielen Ebenen nachweislich existiert. Fabricio Benedetti, ein führender Forscher auf diesem Gebiet, spricht davon, dass es sich wesentlich um „psychosoziale Zusammenhänge von Behandlungen und Patienten“ handle, welche eine entscheidende Rolle für den Behandlungserfolg spielen [1, 2].
Was wir als Ärzte wie und wann sagen, nimmt erheblichen Einfluss auf die Behandlung. Wie ein Mensch im Laufe der Therapie „be-hand-elt“, berührt (oder nicht berührt) wird, macht erhebliche Unterschiede. Ob ein Medikament oral, in Spritzen- oder Infusionsform verabreicht wird, kann dessen Wirkung dosisunabhängig verändern. Farben und Gerüche einer Medikation spielen eine Rolle. Vor allem aber sind die „eigen-sinnigen“ Erfahrungen, Erwartungen und Hoffnungen eines erkrankten Menschen entscheidend. Zu lernen, die jeweilige Summe dieser Faktoren individuell besser einzuschätzen, dies ist für mich eine komplementärmedizinische Frage erster Ordnung.

\section{Soziale, ökologische, kulturelle} und spirituelle Zusammenhänge von Gesundheit sind unverzichtbare Elemente

Gesundheit, Krankheit, Behandlung und Heilung sind keine ausschließliche Domäne des Gesundheitswesens. Sie entwickeln sich als Lebensereignisse und werden dort gefördert, geschützt, bedroht oder geheilt, wo die Menschen leben, arbeiten, lieben, spielen und sich miteinander in ihren Umwelten austauschen. Mitte der 1980er-Jahre durfte ich im Europäischen Büro der WHO an der Entwicklung eines neuen Konzepts zur „Gesundheitsförderung“ mitarbeiten. Diese Arbeit kulminierte 1986 in der „Ottawa-Charter for Health Promotion“, die inzwischen weltweit zu einem wichtigen Leitfaden von Gesundheitspolitik geworden ist. „Gesundheitsförderung“ geht, als Konzept und Stra- gewählte Worte der Ärzte und des Pflegepersonals
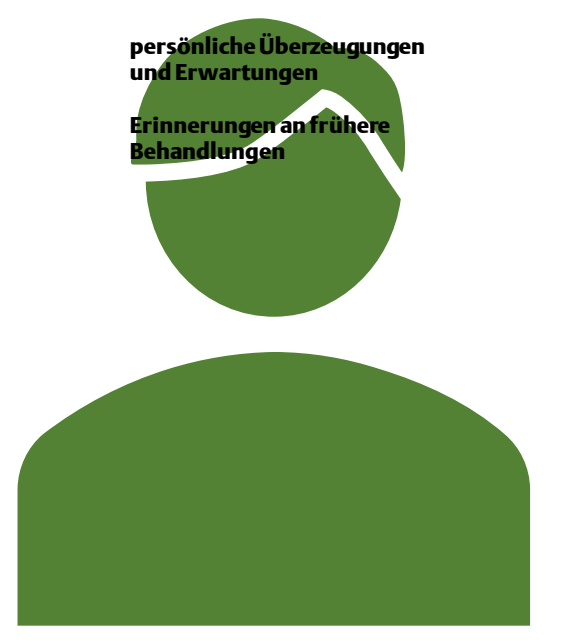

Sichtweisen der behandelnden Ärzte, des Krankenhauses und der verwendeten Instrumente
Farbe, Form, Geruch und Geschmack der Medikamente
Austausch mit anderen Patienten und Menschen 
tegie, über einen medizinisch-krankheitsbezogenen Begriff von Prävention hinaus. Sie betont eine umfassendere, politische, soziale, ökologische, partizipative Sicht von Gesundheit.

Angesichts der schwierigen Abgrenzungen zwischen „Religionen“ und „Spiritualität“ wurde dieser Aspekt in der WHOCharta nicht berücksichtigt. Er ist jedoch, nach meiner Ansicht, ebenfalls essenziell für Gesundheit und Heilung. In diesem Zusammenhang ist manche apodiktische Forderung nach allumfassender Achtsamkeit zu formelhaft. Ich bevorzuge den Ausdruck „weniger achtlos“, da er eine beständige, aber machbare Haltung betont. Ansätze der Gesundheitsförderung sollten in jeder Ausund Weiterbildung sowie in die tägliche Praxis einfließen.

\section{Den Begriff der „Natur“ kritisch überdenken}

Nach meinem Verständnis birgt heutzutage die umstandslose Verwendung von Begriffen wie „Natur“ und „Natürlichkeit“ ein nicht einzulösendes Versprechen. Die Praktiken der modernen Landwirtschaft, Lebensmittelproduktion oder heutige Essgewohnheiten sowie die vorherrschenden Bewegungspraktiken in unseren Arbeitsabläufen, in Transport- und Verkehrswegen oder in Wohnformen verflechten Natur und moderne Technologien aufs Engste miteinander. Diese Entwicklungen müssen wir hinterfragen und, wo möglich, verringern. Unsere Lebensweisen gänzlich durch „natürliche“ Alternativen ersetzen zu können, ist für die meisten Menschen nicht möglich. Als moralischer Anspruch kann diese Betonung viele Menschen auch überfordern.

Es geht auch um persönliche Haltung und die respektvoll angemessene Auswahl von jeweils Wünschenswertem und Machbarem.

Meine Suche nach sich gegenseitig ergänzenden Möglichkeiten von Medizin, Therapie und Gesundheitsförderung ist nicht abgeschlossen. „Ganzheitlichkeit“ ist für mich ein erstrebenswertes Ziel, das ich nie endgültig erreichen werde. Ich kann mich diesem Ziel aus biografischer, pathophysiologischer, anatomischer, energetischer, spiritueller, psychologischer Sicht oder anderen Sichtweisen immer nur annähern. Als Arzt oder Therapeut kann ich nach bestem Wissen und Gewissen, ethisch bewusst, möglichst in Zusammenarbeit mit den betroffenen Menschen, handeln und behandeln. Heilen bedeutet mehr als medizinisch (be)handeln.

„Primum non nocere, secundum cavere, tertium sanare.“ - „Erstens nicht schaden, zweitens vorsichtig sein, drittens heilen.“

Ganz gleich welche Theorien, Methoden oder Techniken wir anwenden, dieser antike Wahlspruch der Heilkunde bleibt wegweisend. „Nicht schaden“ heißt auch bisweilen, abwarten zu können, auf unnötige Interventionen zu verzichten oder sich, zusammen mit den betroffenen Menschen, respektvoll die Grenzen des Möglichen einzugestehen.

Interessenkonflikt: Der Autor erklärt, dass keine Interessenkonflikte bestehen.

\section{Online zu finden unter}

http://dx.doi.org/10.1055/a-1163-5502

\section{Literatur}

1 Benedetti F, Piedimonte A. The neurobiological underpinnings of placebo and nocebo effects. Semin Arthritis Rheum 2019; 49 (3S): S18-S21

$\overline{2}$ Benedetti F, Amanzio M. The placebo response: How words and rituals change the patient's brain. Patient Education and Counseling 2011; 84 (3): 413-419

$\overline{3}$ Farber B et al. Choosing psychotherapy as a career. J Clin Psychol 2005; 8: 1009-1031

$\overline{4}$ Frank J. Die Heiler. Wirkungsweisen psychotherapeutischer Methoden. Stuttgart: Klett-Cotta; 1981

$\overline{5}$ Fuchs T. Das Gedächtnis des Leibes. Locumer Pelikan 2012; 3: 103-106

$\overline{6}$ Hinshelwood RD. Editorial. Int ] Therapeutic Communities 1992; 4: 167-168

$\overline{7}$ jäeggi E. Und wer trainiert die Therapeuten? München: DTV; 2004

$\overline{8}$ Lakoff G, Johnson M. Leben in Metaphern: Konstruktion und Gebrauch von Sprachbildern. Heidelberg: Carl Auer; 2011

$\overline{9}$ Milz H. Ganzheitliche Medizin. Neue Wege zur Gesundheit. Königsstein: Athenäum; 1985

$\overline{10}$ Milz H. Der wiederentdeckte Körper. Vom schöpferischen Umgang mit sich selbst. Zürich/München: Artemis und Winkler; 1992

$\overline{11}$ Milz H. Der eigen-sinnige Mensch. Körper, Leib und Seele im Wandel. Aarau/München: AT; 2019

$\overline{12}$ Sarasin P. Der öffentlich sichtbare Körper. In: Sarasin P, Tanner J, Hrsg. Physiologie und industrielle Gesellschaft. Frankfurt a.M.: Suhrkamp; 1998

$\overline{13}$ Schipperges $\mathrm{H}$. Medizin an der Jahrtausendwende: Fakten, Trends, Optionen. Frankfurt: Josef Knecht; 1991: $312 f$ $\overline{14}$ Valery P. Ich grase meine Gehirnwiese ab. Frankfurt: Büchergilde Klassik; 2011

$\overline{15}$ Whitmont E. Die Alchemie des Heilens. Göttingen: Burgdorf; 1993

$\overline{16}$ World Health Organization: Ottawa Charter for Health Promotion. 1986; Im Internet: http:// www.euro.who.int/_data/assets/pdf_ file/0006/129534/Ottawa_Charter_G.pdf?ua=1

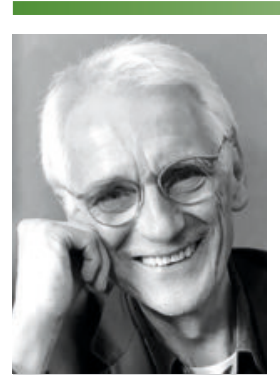

Prof. (em.) Dr. med. Helmut Milz

Marquartstein, Bayern

helmut.milz@gmail.com

Helmut Milz studierte Medizin und Soziologie an der FU Berlin und ist Arzt für psychosomatische Medizin, Psychotherapie und Allgemeinmedizin; seit 1985 Studien von „ganzheitlich/integrativer Medizin“, postgraduierte Studien der Public Health (UC Berkeley), Weiterbildungen in Leibund Körpertherapien, TCM. Er war Inhouse-Consultant der WHO für Gesundheitsförderung und Oberarzt in der Psychosomatik, von 1994-2018 kassenärztliche Praxis, emeritierter Honorarprofessor für Public Health, Universität Bremen; internationale Seminar- und Vortragstätigkeiten; Buchautor, zuletzt „Der eigen-sinnige Mensch“. Körper, Leib und Seele im Wandel (2019). 